Daniel Ricardo Calderón Ramírez Candidato a Magíster en Planificación y Gestión del Turismo Candidato a Doutorado en Planejamento y Gestão do Território, Universidad Federal do ABC, Sao Paulo, Brasil. Magíster en Desarrollo Rural. [danielcalderon137@hotmail.com]

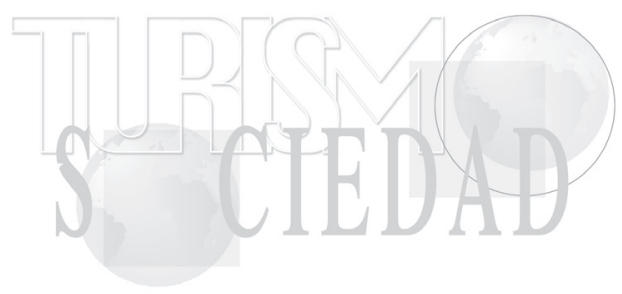

\section{LA PARTICIPACIÓN COMO BASE DEL TURISMO COMUNITARIO Y EL MANEJO DE LOS RECURSOS DE USO COMÚN. SENDERO DE RITAKUWAS, SIERRA NEVADA DE EL COCUY, GÜICÁN Y / O CHITA, BOYACÁ, COLOMBIA ${ }^{1}$}

1 Tesis de grado para optar al título de Magíster en Desarrollo Rural de la Pontificia Universidad Javeriana, Bogotá, Colombia. Dirigida por Humberto Rojas Pinilla, docente investigador de la Facultad de Estudios Ambientales y Rurales.

Fecha de recepción: 23 de mayo 2014.

Fecha de modificación: 30 de julio de 2014

Fecha de aceptación: 11 de agosto de 2014.

Para citar el artículo: Calderón, D. (2014). La participación como base del turismo comunitario y el manejo de los recursos
PARTICIPATION AS A BASE OF COMMUNITY TOURISM

AND THE MANAGEMENT OF COMMON POOL RESOURCES. RITAKUWAS TRAIL, SIERRA NEVADA DE EL COCUY, GÜICÁN AND CHITA, BOYACÁ, COLOMBIA

\section{Resumen}

El objetivo principal de este estudio fue analizar el sendero que conduce al Ritakuwa Blanco, montaña de 5.330 m. s. n.m. ubicada en la Sierra Nevada de El Cocuy, Güicán y / o Chita, como un recurso de uso común (RUC). A través de este RUC se han establecido servicios turísticos y se han dado diferentes formas de participación por parte de las comunidades locales rurales, quienes lo usan y aprovechan. Por tanto, es necesaria una acción colectiva de los usuarios capaz de formular acuerdos o crear instituciones para el autogobierno y el uso sostenible del RUC, con el fin de evitar la tragedia de los bienes comunes ${ }^{2}$. Los prestadores de servicios turísticos decidieron participar en la planificación del ecoturismo debido a los estímulos y beneficios que pudieran llegar a recibir, como el fortalecimiento de la acción colectiva, la mayor autonomía y autogobierno para la sostenibilidad del sendero, la formalidad en la cadena de valor del turismo y la diversificación de los atractivos turísticos.

Palabras clave: recursos de uso común, instituciones, autogobierno, acción colectiva, participación, Sierra Nevada de El Cocuy, Güicán y / o Chita.

\footnotetext{
de uso común. Sendero de Ritakuwas, Sierra Nevada de El Cocuy, Güicán y / o Chita, Boyacá, Colombia, Anuario Turismo y Sociedad, vol. XV, pp. 17-29. DOI: 10.18601/01207555.n15.02 2 HARDIN (1968) utiliza esta expresión que simboliza la degradación del ambiente que puede esperarse siempre que muchos individuos utilizan al mismo tiempo un recurso común. Cada individuo se encuentra atrapado en un sistema que lo compele a aumentar su ganancia sin ningún límite para un recurso que es limitado.
} 


\section{Abstract}

The main objective of this study was to analyze the trail that leads to Ritakuwa Blanco, mountain $5,330 \mathrm{~m}$, located in the Sierra Nevada de El Cocuy Güicán and / or Chita, as a common resource. Through this common resource tourist services have been established, giving different forms of participation by local rural communities. Therefore, is necessary a collective action by users, able to formulate agreements or self-government institutions towards sustainable use of the common resource, in order to avoid the tragedy of the commons. The decision of the tourism service to participate in the planning of ecotourism has been understood since the incentives and benefits that can receive as: strengthening collective action, greater autonomy and self-government for the sustainability of the trail, the formality tourism value chain and diversification of tourist attractions.

Keywords: common resources, institutions, self-government, collective action, participation, Sierra Nevada Cocuy, Güicán and / or Chita.

\section{Introducción}

En Boyacá, Colombia, al norte de la cordillera oriental, entre los municipios de El Cocuy y Güicán de la Sierra, se encuentra la zona de recreación general exterior ${ }^{3}$ del Parque Nacional Natural El Cocuy, representada por uno de los destinos turísticos de alta montaña más importantes en Colombia: La Sierra Nevada de El Cocuy, Güicán y / o Chita. En ella se encuentra el sendero de Ritakuwas, única vía de acceso hacia el glaciar de la montaña nevada más alta de la Sierra Nevada: el Ritakuwa Blanco (5.330 m. s.n. m.).

3 Corresponde a aquellas áreas que por sus condiciones naturales le ofrecen al visitante la posibilidad de recreación al aire libre (PNN EL COCUY, 2011a).
El sendero transita por la cuenca del río Playitas, afluente que nace por el descongelamiento del glaciar y se alimenta de la escorrentía acumulada en el páramo donde yace la cuenca. Las características ecológicas del sendero permiten deducir que es un recurso que debe ser usado de forma limitada, restrictiva y responsable.

Alrededor del sendero, las comunidades campesinas han organizado la prestación de servicios turísticos a visitantes pasadías ${ }^{4}$, facilitándoles el acceso al borde del glaciar. Los servicios de guías, alquiler de caballos, alimentación y alojamiento son las principales fuentes de ingresos para algunas familias campesinas que en cada temporada alta (diciembre / febrero, Semana Santa y junio / julio) reciben en promedio más de diez mil turistas en el parque (CALDERÓN, 2007).

Cada prestador de servicios accede al sendero con la ayuda de un caballo, y cada animal usa una unidad de recurso; esta se refiere al espacio que ocupa el caballo. Las unidades de recurso disponibles (flujo) responden a la capacidad de carga turística (CCT) que tiene el sendero ( sistema de recurso), la cual es limitada.

Ya que el sendero es de libre acceso para la comunidad local prestadora de servicios, este se puede catalogar como un recurso de uso común (RUC) entendido como:

Un sistema de recursos naturales o hechos por el hombre que es lo suficientemente grande como para volver costoso (pero no imposible) excluir a destinatarios potenciales de los beneficios de su uso [...] El acceso a un RUC puede limitarse a un solo individuo o empresa, o bien a múltiples individuos o grupos de individuos que usan el sistema

4 Catalogados como turistas no experimentados para la práctica del montañismo. Su falta de conocimientos y aclimatación a las condiciones atmosféricas de la montaña les impiden subir hasta el borde del glaciar por sí sólos, por esta razón alquilan caballos. 
de recursos al mismo tiempo. (Ostrom, 2004, p. 66)

Siguiendo a Plott (1979), el proceso de sustracción o uso del recurso se denomina apropiación, por lo tanto, los prestadores de servicios son apropiadores del sistema de recurso sendero. Por otro lado, los actores que se encargan de garantizar que el sistema de recursos siga manteniendo su función se denominan proveedores, quienes aseguran que las condiciones del sendero sean propicias y seguras para seguir utilizándolo. En el caso del sendero de Ritakuwas, la apropiación y provisión del sendero se da gracias a las acciones emprendidas por los apropiadores, quienes al mismo tiempo son proveedores.

Esta relación de apropiación y provisión lleva a concluir que los usuarios de un RUC deben tener condiciones de autogobierno, pues las condiciones y decisiones de apropiación y provisión deben darse de manera participativa y consensuada para evitar la sobreexplotación o la figura que E. Ostrom (2004) define como: free rider o gorrón, que se beneficia del esfuerzo de otros en las acciones de provisión. Para evitar esto se establece un conjunto de instituciones, acuerdos, normas o pactos que controlan las acciones de los prestadores de servicios, regulando las "reglas del juego, que transforman elementos clave implicados en la decisión de un individuo" (CÁRDENAS ET ÁL. 2004, p. 92).

Las soluciones de un autogobierno eficaz y eficiente demuestran el grado de participación que la comunidad tiene sobre el uso y manejo del RUC (CÁRDENAS, 1995, 2001, 2009). Ello demuestra que, en el caso del turismo comunitario, es fundamental identificar la manera en que las comunidades locales se involucran en el manejo de los recursos tomando decisiones consensuadas que permitan generar instituciones confiables que hagan posible el autogobierno.
La efectividad de las instituciones, como la capacidad de carga turística (CCT), los límites de cambio aceptable (LAC) o el monitoreo de impactos, se logra por medio de una coherencia entre los beneficios obtenidos, el esfuerzo invertido en alcanzarlos y la credibilidad en mantenerlos. "La elección de conducta de un individuo en cualquier situación particular depende del modo como este conozca, considere y evalúe los costos y beneficios de las acciones, así como de la percepción de su vínculo con resultados que también incluyen una mezcla de beneficios y costos" (OSTROM, 2004, p. 70).

Sin embargo, se reconoce que existe un involucramiento pasivo ${ }^{5}$ de los prestadores de servicios turísticos del sendero de Ritakuwas en la toma de decisiones para el control, manejo y provisión del RUC. No existe una acción colectiva porque no hay una participación para la asignación de responsabilidades que generen acuerdos, o instituciones que permitan la provisión eficaz para el uso del sendero. Así, los arreglos físicos del sendero y cualquier estrategia de disminución de impactos negativos no son asumidos por todos los usuarios. Este es un ejemplo de la tragedia de los bienes comunes, pues cada prestador de servicios turísticos, articulado a una cadena de valor, trata de sacar el mayor provecho del sendero sin límites en el uso.

De este modo, en la investigación se plantea la siguiente pregunta ¿cómo los prestadores de servicios turísticos toman decisiones acerca del uso del sendero de Ritakuwas y cómo la participación crea soluciones de autogobierno que puedan incrementar su uso sostenible? Para responder fue necesario identificar las interacciones e instituciones de la comuni-

5 Según C. Ashley (1995), el involucramiento pasivo en una actividad turística se reconoce cuando un grupo de individuos locales se limita a la simple prestación del servicio y a la venta de recursos. 
dad en el RUC: a) caracterizando los atributos ecológicos y de infraestructura del sendero; b) identificando las relaciones socioculturales de los usuarios frente al recurso; y c) reconociendo las instituciones de control y manejo del sendero y la participación de la comunidad en su creación y consenso.

\section{Metodología}

La propuesta metodológica construida para resolver la pregunta de investigación utilizó como eje principal el enfoque participativo por medio de herramientas participativas (GeILfus, 2002). Se usaron cuatro técnicas para el levantamiento de la información: técnicas de grupo, diagnóstico participativo, observación de campo, entrevista y comunicación oral. Los instrumentos de dichas técnicas fueron: a) diálogo y entrevista semiestructurada con informantes clave y grupos de prestadores de servicios; b) observación participante en actividades como recorridos, salidas de campo, reuniones y eventos; y c) identificación del árbol de problemas para hacer un diagnóstico participativo. Se utilizaron otras metodologías como la Guía metodológica de facilitación en cadenas de valor (GTZ, 2009), el pentágono de activos del enfoque de medios de vida sostenible (DFID, 2004) y la metodología para la determinación de la capacidad de carga turística propuesta por Parques Nacionales (PNN EL CocuY, 2011b).

\section{Resultados}

El sendero de Ritakuwas es la vía de acceso hacia un atractivo turístico de la Sierra Nevada de El Cocuy, Güicán y / o Chita: el glaciar del Ritakuwa Blanco (5.330 m. s. n. m.). El sendero fue abierto por los primeros campesinos que habitaron el sector de Ritakuwas antes del establecimiento del PNN El Cocuy y del desarrollo del turismo en la región. Tiene una longitud de $6.800 \mathrm{~m}$ desde su inicio (punto donde es posible llegar en carro a una altura de 3.800 m. s. n. m.) hasta el borde del glaciar (4.800 m. s. n. m.). El ancho del sendero varía entre 2 y $20 \mathrm{~m}$. Presenta problemas de filtración de agua y compactación del suelo por acción del tránsito de caballos $\mathrm{y}$ personas.

El sendero trascurre en un $60 \%(4.080 \mathrm{~m})$ por el ecosistema de páramo húmedo (PNN El CocuY, 2011b); el otro 40\% (720 m) por el ecosistema de morrena o superpáramo, presente a partir de los 4.350 m. s.n.m. En el páramo se destaca la abundancia poblacional de frailejón Espeletia lopezzi, que posee especies con más de $2 \mathrm{~m}$ de altura. Así mismo:

Predomina el chusque (Clusia sp), también llamado bambú de páramo [...] existe una gran extensión de pajonales como la Calamagrosti efusa [...] Varias especies de frailejón, helecho, cardón y musgos son algunas de las plantas azonales. Así mismo, una gran riqueza de especies endémicas, incluyendo varias especies de frailejón (Espeletia sp) y lítamo (Draba litamo). (PNN El COCUY, 2011a)

Respecto a la fauna, se encuentran especies endémicas como el lagarto collarejo (Stenocercus lache), especies de aves como el cucarachero apolinar (Cistothorus apolinari) y el reintroducido cóndor de los andes (Vutur gryphus) (PNN EL COCUY, 2011b).

El sendero cuenta con una infraestructura turística instalada por El Parque Nacional Natural El Cocuy, que con la ayuda de algunos prestadores de servicios turísticos instaló la señalética, representada tanto por vallas como por mojones ("hombres de piedra"), que indican la orientación del sendero. Así mismo, se han construido un puente y otros cimientos en los pasos necesarios para que puedan transitar los caballos. 
La participación de la comunidad campesina local en el desarrollo del turismo comienza cuando los habitantes, ubicados en las diferentes veredas del municipio de Güicán (El Calvario, El Centro, El Jordán, El Tabor, La Cueva, La Unión, San Ignacio, San Juan, San Luis, San Roque), ven en el turismo una opción económica a la cual se podrían dedicar en los meses de verano, justo cuando no hay actividades agropecuarias para hacer. Así, de manera desagrupada y sin dirección alguna, empezaron a prestar servicios de alojamiento, alimentación y guía, en los cuales cada uno de los prestadores de servicios trabajaba de forma individual. JOAQUÍN VALDERRAMA, funcionario del parque desde hace quince años, explica:

A medida que avanzaba el turismo, la comunidad empezó a ver que sus integrantes tenían objetivos y metas comunes, pero no lograban trabajar juntos por ellas; que el desarrollo del turismo era un arma de doble filo: por un lado traía aparentemente beneficios económicos, pero también de forma mal controlada y manejada estaba causando grandes impactos sobre el medio ambiente, conflictos con el parque y además ocasionaba problemas entre ellos al no poder unificar precios ni servicios. (Entrevista semiestructurada del autor a funcionario del Parque Nacional Natural El Cocuy, 04 de junio de 2010)

Hoy en día, luego de más de cuarenta años de desarrollo del turismo en la región, las comunidades campesinas han especializado la prestación de servicios apoyándose en diferentes actores públicos y privados, como Parques Nacionales, el SENA, la Camára de Comerció de Duitama, la Alcaldía de Güicán de la Sierra, entre otros. Aunque existe una normativa propuesta por la administración del Parque Nacional Natural El Cocuy para el desarrollo del ecoturismo, no ha sido fácil hacerla cumplir por las complejas característi- cas que presenta el sendero, reconocido como un recurso de uso común. AlFredo CORREA, prestador de servicios ecoturísticos desde hace diez años, dice:

Cuando empieza a llegar tanto turista, también llegan muchos problemas, empiezan a aparecer personas de los pueblos que, de un momento a otro, son guías o arrieros sin siquiera conocer bien la Sierra [...] además, entre nosotros mismos nos peleábamos por los turistas; unos lo hacían de forma más descarada porque regalaban prácticamente el trabajo y muchas veces lo hacían de pura envidia para quitarle a uno los clientes [...]. (Entrevista semiestructurada del autor a prestador de servicios ecoturísticos en el Parque Nacional Natural El Cocuy, 11 de junio de 2010)

La comunidad local campesina, según el servicio turístico que presta, se puede caracterizar en tres grupos: 1) Casa de guías, quienes prestan el servicio de alojamiento y alimentación en posadas tipo cabaña o chalet; en la actualidad existen tres: Ritakuwas, Sierra Nevada y Portón de la Sierra. 2) Guías y arrieros, encargados del guionaje de grupos por el sendero hasta el borde del glaciar. Se calcula que puede haber más de cincuenta personas que prestan este servicio, en promedio cada uno de ellos puede tener entre dos y siete caballos. Hay registro de más de trescientos caballos en un día en el sendero. 3) Transportadores, encargados de conducir a los turistas en camionetas privadas desde el casco urbano del pueblo hasta la entrada del parque.

Los diferente prestadores de servicios se articulan dentro de la cadena de valor del turismo a través de las casas de guías, empresas privadas con registro nacional de turismo (RNT), que venden principalmente el servicio de alojamiento y alimentación, pero donde los prestadores como transportadores, guías y arrieros también ofrecen sus servicios. 
La articulación de los servicios conforma paquetes turísticos que son ofrecidos por agencias de turismo mayoristas y operadoras. En el mapeo de la cadena de valor (figura 1) se reconoce la participación de los prestadores de servicios y otros actores, así como las funciones y las relaciones de gobernanza y poder según parámetros políticos y culturales.

\section{Cadena de valor del turismo Ritakuwas}

FIGURA 1. Mapeo de la cadena de valor del turismo Ritakuwas. Fuente propia.

Funciones básicas

\begin{tabular}{|c|c|c|c|c|c|}
\hline $\begin{array}{c}\text { Ofertar: } \\
\text { promocionar } \\
\text { vender }\end{array}$ & Transportar & $\begin{array}{c}\text { Registrar, } \\
\text { control y registro } \\
\text { PNN El Cocuy }\end{array}$ & $\begin{array}{l}\text { Alojamiento y } \\
\text { alimentación }\end{array}$ & $\begin{array}{l}\text { Guionaje } \\
\text { arriería } \\
\text { servicios }\end{array}$ & $\begin{array}{l}\text { Demandar } \\
\text { turista o } \\
\text { visitante }\end{array}$ \\
\hline
\end{tabular}

Mapeo de las subcadenas y gobernanza entre actores, género y número de empresas

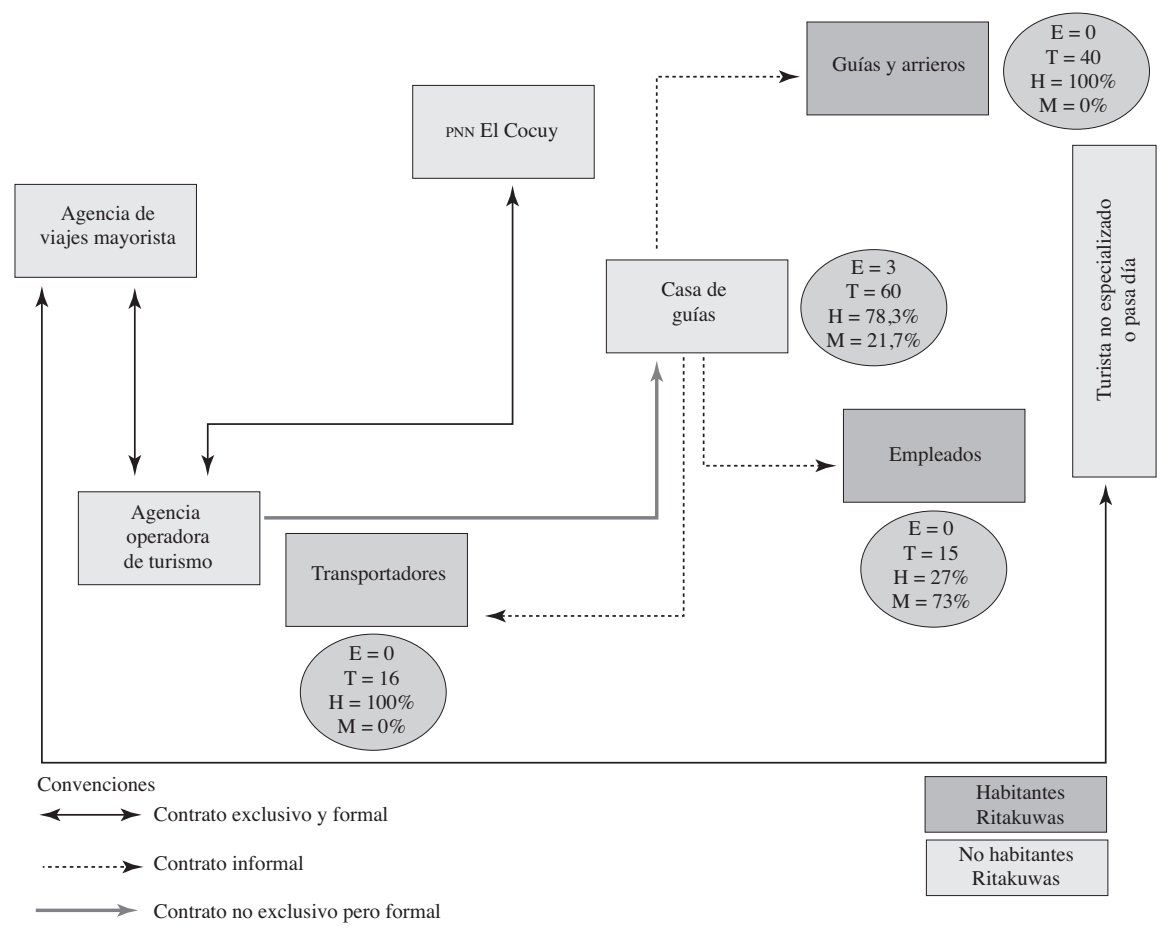

Convenciones

$\mathrm{E}=$ Número de empresas legalmente constituidas .

$\mathrm{T}=$ Número de personas que conforman dicho grupo.
$\mathrm{H}=$ Porcentaje de hombres que conforman dicho grupo.

$\mathrm{M}=$ Porcentaje de mujeres que conforman dicho grupo. 
En la cadena de valor se pueden identificar los tipos de contratos económicos entre los diferentes prestadores de servicios: a) El contrato exclusivo y formal, presente solamente entre las agencias de viajes y el turista. Representa la seguridad laboral y, por ende, económica. b) El contrato informal, que muestra la informalidad en acuerdos, alianzas, pactos y convenios. c) El contrato no exclusivo pero formal, donde no hay una exclusividad en la contratación, pero sí una formalidad, ya que existe un documento legal que constituye un contrato. Se puede observar que la mayoría de contratos son de carácter informal, lo que evidencia la informalidad de la actividad turística. William AlbarRACín, guía de montaña del parque, dice:

El problema más evidente es la falta de coordinación entre los prestadores de servicios. No nos hemos dado cuenta de que estamos perdiendo plata. Mientras las agencias de Bogotá cobran un millón y medio por un tour, nosotros terminamos haciendo todo y ganando menos de la mitad de esa plata [...] Si nosotros nos ponemos las pilas, podemos vender los planes por Internet y hacer el doble de plata [...] A veces lo llaman a uno las agencias de Bogotá, dicen que viene gente para una fecha, uno los espera y al final no salen con nada. A última hora le cancelan y a veces uno ya ha comprado comida y dejado de hacer cosas para poder cumplirles [...] Si uno les pide plata por adelantado nunca la mandan [...] yo he dejado de trabajar con algunas agencias porque no son serias. (Entrevista semiestructurada a guía de alta montaña, Parque Nacional Natural El Cocuy, 11 de febrero de 2011)

La cadena de valor muestra claramente la heterogeneidad de actores y prestadores de servicios turísticos, lo que se traduce en informalidad del turismo por el tipo de contratos informales; cualquier persona con un caballo puede llegar al sendero y alquilarlo, no hay reglas claras sobre quiénes y bajo qué condiciones pueden prestar ese servicio. Así mismo, hay desigualdad en los beneficios para algunos grupos de actores, sobre todo en cuanto a precios y estabilidad de la actividad. También existe desigualdad de género en la participación del turismo, ya que la mayoría de prestadores de servicios son hombres, no hay mujeres guías. Finalmente, hay relaciones jerárquicas y de subordinación con ausencia de participación de algunos actores. LUIS EDUARDO BARÓn, guía y arriero desde hace cinco años, explica:

Realmente yo no participo en las reuniones que hace Parques para la regulación del turismo porque aquí no hay un control claro de la actividad. Uno no tiene claro quiénes son arrieros y quiénes no. Se supone que uno presta un servicio porque ha recibido las charlas de Parques Nacionales, el SENA [...] pero llegan otros aparecidos y hacen lo mismo más barato y a veces ni siquiera llevan a la gente por donde es porque no conocen. ¿Entonces qué control hay? Yo he pasado la queja a Parques Nacionales y ellos me dicen que eso es problema de nosotros. Para qué participar si no nos escuchan. (Entrevista semiestructurada del autor a prestador de servicios ecoturísticos, guianza y arriería en el Parque Nacional Natural El Cocuy, 10 de febrero de 2011)

Así mismo, la cadena de valor evidenció que los habitantes de Ritakuwas, entre quienes se encuentran los integrantes de las casas de guías, los guías-arrieros y los transportadores, participan en la manutención y provisión del sendero. Durante dos o tres días se conforman grupos para realizar tareas de remoción de piedras, asentamiento de tierra, mantenimiento de mojones y señales, y arreglos del sendero en general, que incluyen recoger basura. Los demás actores (no habitantes de Ritakuwas), como las agencias mayoristas y operadoras, no participan de ninguna actividad de provisión y mantenimiento del sendero. DAIRON LEÓn, habitante de la vereda La 
Capilla, quien vende servicios de alojamiento, alimentación y alquiler de caballos, dice:

Está bien que las agencias de Bogotá y Medellín nos manden turistas, pero a veces se demoran en pagar [...] Una vez se demoraron un mes en que me pagaran un tour y uno sí debe pagar ahí mismo todo lo que se le ofrece al turista [...] Otra cosa que no me gusta es que ellos ganan más del doble de lo que uno gana, y fuera de eso, cuando uno les pide un aumento dicen que otros les dan los servicios más baratos. También, cuando uno les dice que hay convites para arreglar el sendero y que se necesita dinero, ellos se hacen los de la vista gorda y no mandan ni un centavo. El arreglo del sendero lo hacemos nosotros y otros ganan. (Entrevista semiestructurada del autor a prestador de servicios ecoturísticos en el Parque Nacional Natural El Cocuy, 10 de febrero de 2011)

Por medio de un diagnóstico participativo, que emplea la herramienta árbol de problemas (figura 2), los prestadores de servicios reconocen que el desarrollo del turismo en el sector de Ritakuwas es insostenible. Se identificaron las causas y las consecuencias de dicha insostenibilidad y se concluyó que la peor consecuencia es que la administración del Parque Nacional Natural El Cocuy prohíba el turismo por ir en contravía de sus objetivos de conservación.

\section{Diagnóstico participativo árbol de problemas}

FIGURA 2. Diagnóstico participativo árbol de problemas

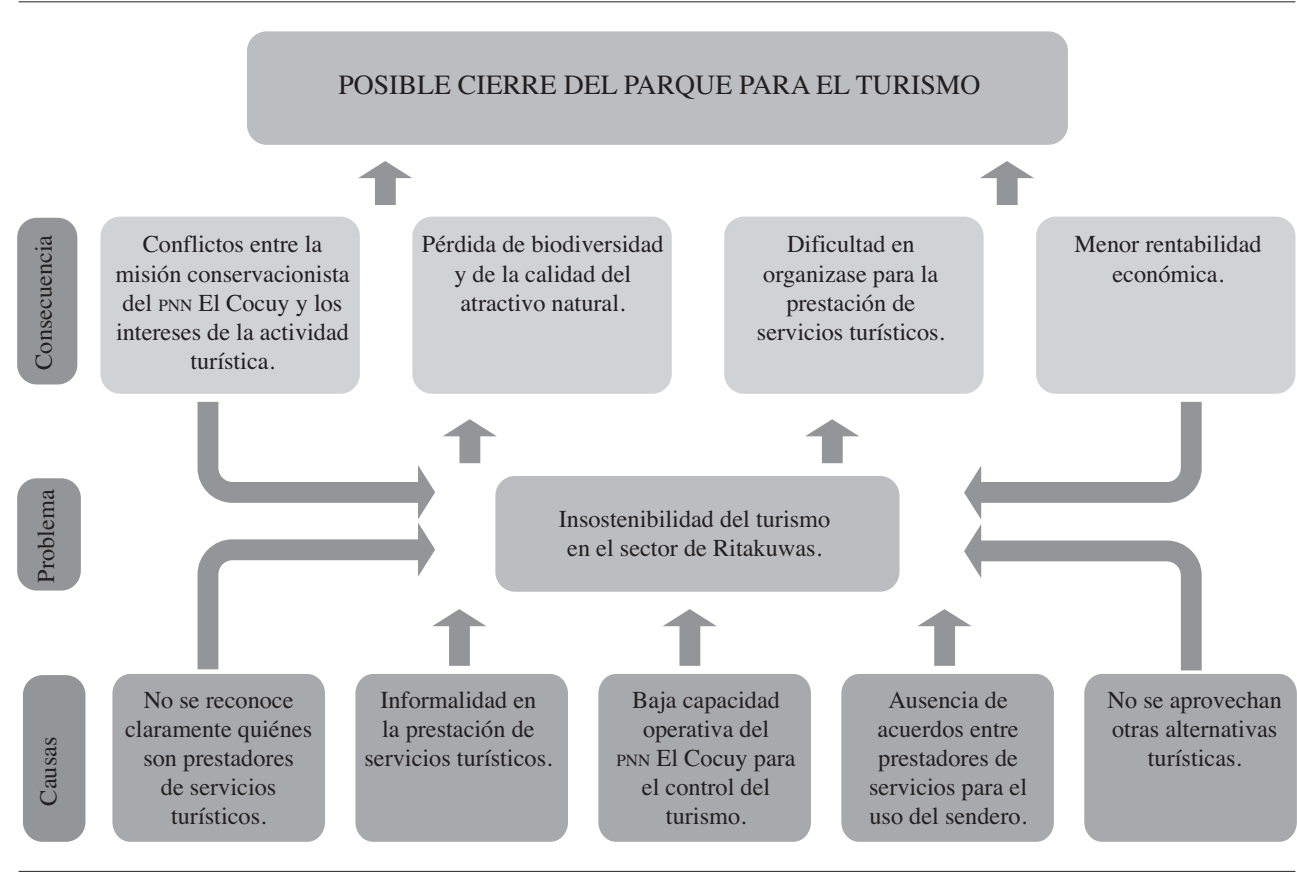

Fuente: Elaboración del autor. 
Frente a la problemática reconocida por la comunidad prestadora de servicios, esta decide hacer parte de un proceso de ordenamiento del ecoturismo que adelanta la administración del PNN El Cocuy con el apoyo de la agencia estadounidense para el desarrollo USAID (PNN EL CocuY, 2011c). En consecuencia, la comunidad participa en un proceso de aprendizaje y capacitación que le permite ser parte de un equipo de trabajo junto a funcionarios y contratistas del parque para el establecimiento de un plan de ordenamiento del ecoturismo (POE) que incluye: a) diagnóstico general del área y de la actividad turística; b) ordenamiento y zonificación del área con componentes como capacidad de carga turística, límites de cambio aceptable y reglamentación de la actividad turística; y c) plan de acción para el desarrollo del ecoturismo. LIGIA NUMPAQUE, contratista del parque, explica:

La participación de los prestadores de servicios en el POE permitió que este fuera más eficaz, que se reconociera a la gran mayoría de prestadores de servicios y se lograra una articulación entre Parques y la comunidad local. Hasta ahora Parques y la comunidad local prestadora de servicios no habían trabajado en una estrategia conjunta para el ordenamiento del ecoturismo. Que la comunidad local pudiera participar de esta experiencia permitió que el POE no fuera un diagnóstico técnico en el que no se tuviera en cuenta la visión de la comunidad local [...] Las medidas, como capacidad de carga turística, van a ser más efectivas porque fue la comunidad local la que tomó los datos y entendió la necesidad de implementarlas, aunque pueden ser restrictivas y autoritarias si se imponen sin ningún consenso o participación. (Entrevista semiestructurada del autor a contratista del Plan de Ordenamiento Ecoturístico en el Parque Nacional Natural El Cocuy, 31 de marzo de 2011)

Con la participación de la comunidad en el desarrollo del POE se logró identificar y convocar a más del $90 \%$ de prestadores de servicios turísticos, ampliando su reconocimiento con miras a una eventual conformación de una organización social o asociación que permita articularlos. Así mismo, más de 70 prestadores de servicios llevaron a cabo acciones de mejoramiento de la infraestructura física del sendero, como arreglo de puentes, drenajes, señalética, etc. También identificaron que la mejor estrategia para alivianar el uso excesivo del recurso sendero, sin afectar la rentabilidad económica, es generar otros atractivos turísticos complementarios como: senderos ecológicos hacia otros destinos, talleres de esquileo de ovejas y procesamiento de la lana, elaboración de artesanías, talleres de amasijos, entre otras actividades que permiten aumentar los ingresos económicos para la comunidad, así como la participación e igualdad de género en la cadena de valor.

Adicionalmente, la comunidad estableció reglas como el pago de impuestos para la manutención del sendero, el uso de bozales y pañales para los caballos, y la generación de un fondo económico común que permita realizar arreglos periódicos en el sendero. De esta manera la comunidad participa en el diseño de estrategias para reducir los impactos ecológicos negativos haciendo reparaciones en la infraestructura física y proponiendo instituciones de uso que generen una provisión participativa del sendero. Con estas medidas se amplía la articulación entre la administración del Parque Nacional Natural El Cocuy y los prestadores de servicios turísticos hacia la consecución de un ecoturismo que no vaya en contravía de los objetivos de conservación del parque y amplíe la participación de la comunidad local campesina.

\section{Conclusiones}

El sendero de Ritakuwas, que conduce a la Sierra Nevada de El Cocuy, es un recurso de uso común para la comunidad campesi- 
na local prestadora de servicios turísticos. Alrededor de este RUC es posible integrar a la comunidad en igualdad de condiciones de participación, promoviendo una acción colectiva capaz de articular actores en la búsqueda de un bien común (CÁRDENAS, 1995, 2001, 2009).

La participación en una acción colectiva para el autogobierno de un RUC depende de los beneficios recibidos. En este caso, un ejemplo son los procesos de autogobierno, como el arreglo de caminos, la implementación de nuevos atractivos y la reglamentación para el manejo de caballos, propuestas en las cuales la comunidad trabaja conjuntamente con el objetivo de especializar el turismo y sacar mayor beneficio.

C. Ashley postula que la mayor participación de la comunidad local en procesos de desarrollo turístico se obtiene "manteniendo el acceso a los recursos y promoviendo la gestión colectiva" (ASHLEY, 1995, p. 36). La acción colectiva para el desarrollo turístico con comunidades locales debe ser impulsada por los recursos de uso común que fortalecen los lazos entre los individuos que desean alcanzar el mismo objetivo.

La participación de la comunidad local en el proceso de ordenamiento del turismo, propuesta por el PNN El Cocuy, permitió establecer un mecanismo de comunicación entre los prestadores de servicios, pues cuando se les permite a los usuarios comunicarse, obtienen beneficios conjuntos sustancialmente mayores (Ostrom, 2004, p. 11) De esta forma, es posible una autogestión del sendero una vez se han resuelto los problemas de provisión del recurso y de credibilidad entre los prestadores de servicios.

La capacidad de carga turística (CCT) es un cálculo técnico basado en las características biofísicas del sendero y la relación entre visitantes y organismos de control y manejo del turismo (Ministerio DE COMERCIO, INDUSTRIA Y TURISMO, MINISTERIO DE AMBIENTE, VIVIENDA Y DESARROLlo TERRITORIAL, 2006). Más allá de ser un cálculo limitante, puede llegar a ser una estrategia alrededor del autogobierno y la gestión sostenible del turismo que genera acciones de provisión para el manejo de recursos de uso común.

Por lo tanto, la generación de instituciones como la CCT, entre otras que se propusieron, en las cuales se da una coherencia entre los beneficios obtenidos por el RUC y el esfuerzo invertido en alcanzarlos (OSTROM, 2004, p. 69), responde a una necesidad de poder confiar en las acciones que cada prestador de servicios realiza según reglas que se han establecido de manera conjunta. De esta manera:

la elección de conducta de un individuo en cualquier situación particular depende del modo como este conozca, considere y evalúe los costos y beneficios de las acciones, así como de la percepción de su vínculo con resultados que también incluyen una mezcla de beneficios y costos. (OsTROM, 2004, p. 70)

Las acciones de provisión sobre el sendero se presentan no sólo por la necesidad de control del recurso, también por el interés de aumentar la rentabilidad económica, incentivar la innovación de nuevos atractivos turísticos, elevar la calidad en los servicios y fortalecer y generar nuevas instituciones en la cadena de valor del turismo; todo esto sin aumentar los límites en la CCT ni masificar el turismo.

Por tanto, los prestadores de servicios pueden llegar a ser funcionarios del control y del desarrollo del RUC, siempre y cuando se logre organizar a la comunidad alrededor de una acción colectiva (CÁRDENAS, 1995). El manejo de los recursos de uso común puede ser agenciado bajo los principios de participación, libertad, autonomía y empoderamiento de la comunidad local, generados por la credibilidad y confianza de las instituciones (CÁRDENAS, 
1995, 2001, 2009; OSTROM, 2004; CÁRDENAS y OSTROM, 2004; BASURTO Y OSTROM, 2009).

En conclusión, la participación en el turismo comunitario donde existe un RUC puede definirse como una acción conjunta, de un grupo de usuarios de un recurso, hacia la consecu- ción de beneficios mutuos. En ella se establece una organización que busca relacionar las acciones que cada individuo realiza para su propio bienestar, pero que al mismo tiempo deben contribuir al bienestar de los demás. De allí que se vea como una acción colectiva (BERDEGUE, 2000).

\section{Anexos}

FIGURA 3. Instituciones y reglas. Medida de manejo para que el caballo no se coma la vegetación del páramo. Se propone el uso de bozales. Fuente propia.
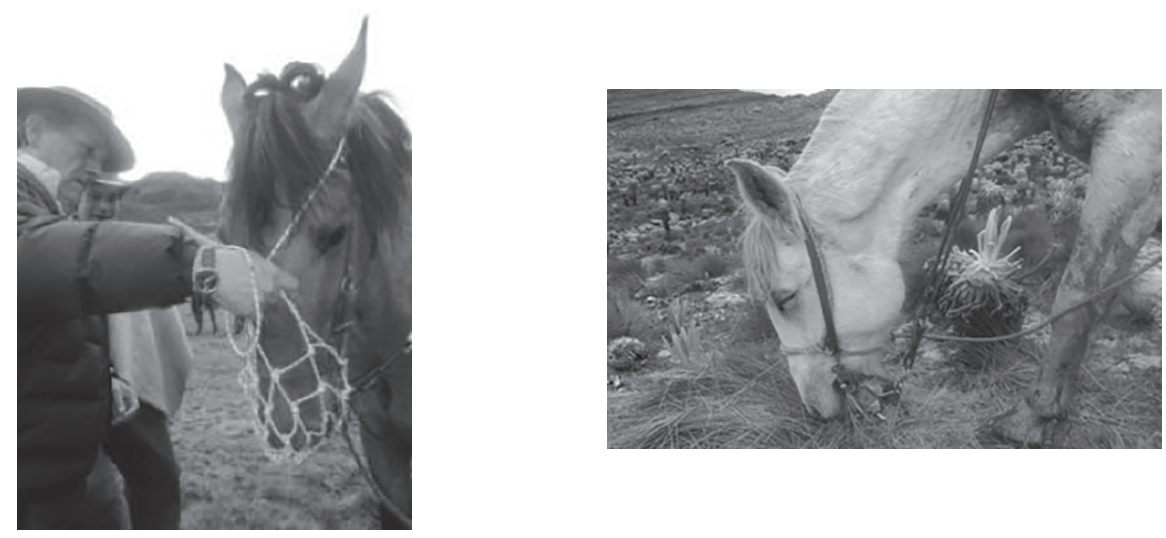

\section{Bibliografía}

Ashley, C. (1995). Tourism, Communities, and the Potential Impacts on Local Incomes and Conservation. Research Discussion Paper 10, Namibia: Directorate of Environmental Affairs, Ministry of Environment and Tourism.

Basurto, X., Ostrom, E. (2009). The Core Challenges of Moving Beyond Garret Hardin. Journal of Natural Resources Policy Research, 1(3), 255-259.

Berdegue, J. (2000). Cooperando para competir. Factores de éxito de las empresas asociativas campesinas. Red internacional en metodologías de investigación en sistemas de producción. Dispo- nible en http:// www.asesoriasparaeldesarrollo.cl/ secciones/documentos/berdegue.pdf, consultado el 6 de octubre de 2009.

CALDERón D. R. (2007). Caracterización de actores sociales turísticos del Parque Nacional Natural El Cocuy, Boyacá-Colombia. Tesis de grado para optar al título de Ecólogo. Bogotá: Pontificia Universidad Javeriana.

CÁRDEnAs, J. (1995). Descentralización y ambiente: construcción de capacidad municipal para la gestión ambiental local en Colombia. Revista Nómadas, 3(septiembre), Fundación Universitaria Central. 
CÁRdenAS, J. (2001). El Sistema Nacional Ambiental (SINA) desde la perspectiva del policentrismo y el neoinstitucionalismo. Revista Javeriana, 647(136).

CÁrdenas, J; Ostrom, E. (2004). “¿Qué traen las personas al juego? Experimentos de campo sobre la cooperación en los recursos de uso común". Desarrollo y Sociedad, 54 (septiembre), 87-132, Universidad de los Andes.

Cárdenas, J. (2009). Dilemas de lo colectivo. Instituciones, pobreza y cooperación en el manejo local de los recursos de uso común. Bogotá: Universidad de los Andes. Colección Cede 50 años.

Correa, A. (2010, junio 11). Entrevista semiestructurada, prestador de servicio ecoturístico Parque Nacional Natural El Cocuy.

DEPARTMENT FOR INTERNATIONAL DEVELOPMENT (DFID). (2004). Hojas orientativas sobre los medios de vida sostenibles, Departamento para el Desarrollo Internacional, Reino Unido.

Deutsche Gesellschaft Für Technische ZuSAMMENARBEIT (GTZ). (2009). Guía metodológica de facilitación en cadena de valor. Lima: Empresa Editora El comercio S.A.

GEILFUS, FRANS. (2002). 80 herramientas para el desarrollo participativo: diagnóstico, planificación, monitoreo, evaluación. San José: Instituto Interamericano de Cooperación para la Agricultura IICA.

HARDIN, G. (1968). The Tragedy of the Commons. Science 162, 1243-1248.

LEÓN, D. (2011 , marzo 31). Entrevista semiestructurada, prestador de servicio ecoturístico Parque Nacional Natural El Cocuy.
MinisTERIO DE COMERCIO, INDUSTRIA Y TURISMO, Ministerio de AMBIENTE, Vivienda y DesarRoLLO TERRITORIAL. (2006). Lineamientos para el ecoturismo comunitario en Colombia.

Numpaque, L. (2011, marzo 31). Entrevista semiestructurada, contratista Plan de Ordenamiento Ecoturístico, Parque Nacional Natural El Cocuy.

Ostrom E. (2004). El gobierno de los bienes comunes. La evolución de las instituciones de acción colectiva. México: Universidad Autónoma de México. Centro Regional de Investigaciones Multiplidisciplinarias, Fondo de Cultura Económica.

PlotT, C. R. (1979). The Application of Laboratory Experiemental Methods Public Choise. En C.S Russel (ed.). Collective Decision Making: Applications from Public Choise Theory. Baltimore: Johns Hopkins University Press.

PNN El Cocuy. (2011a). Plan de Ordenamiento Ecoturístico para la Sierra Nevada de El Cocuy, Güicán y Chita. Boyacá: Parque Nacional Natural El Cocuy.

PNN El Cocuy. (2011b). Determinación de la capacidad de carga aceptable del costado occidental del Parque Nacional Natural El Cocuy (Boyacá) Colombia. Unidad Administrativa Especial del Sistema de Parques Nacionales Naturales de Colombia, Bogotá.

PNN El CocuY. (2011c). Guía metodológica para el monitoreo de los impactos del ecoturismo y determinar la capacidad de carga aceptable en el Parque Nacional Natural El Cocuy Boyacá, Colombia, Bogotá.

VAlDERRAMA, J. (2010, junio 4). Entrevista semiestructurada, funcionario Parque Nacional Natural El Cocuy. 